\title{
A Device Based on Adaptive Filtering Algorithm for Detecting ECG Signal in Motion Artifacts
}

\author{
Liangguang Peng ${ }^{a}$, Tong Bai ${ }^{b}$, Yu Pang ${ }^{c, ~}{ }^{*}$, Guoquan Li, Zhangyong Li \\ School of the Chongqing University of Posts and Telecommunications, Chongqing 400065, China \\ apeng_lg@163.com, bbaitong03@126.com, , ,c 120840727@qq.com
}

Keywords: Detection device, three axis acceleration sensors, adaptive filter, heat rate.

\begin{abstract}
Since the detection of electrocardiogram (ECG) is significant for monitoring the stress and diseases of Policeman and current methods for monitoring ECG in motion have drawback of low accuracy, a system for monitoring ECG signal is presented within this paper. The conductive silica gel is used as the collecting electrode, and a low-power-consumption device for detecting ECG signal have been designed. The movement of human body is sensed by the three-axis acceleration sensor, and variable step-size least mean square adaptive algorithm is proposed by using acceleration signal as reference signal. Through adaptive filter and real-time examining R waves of the ECG signal, heart rate is measured in the movement of human body. The results indicate that detection device can output real-time ECG signal and the maximum error of heart rate is within 4\%, thus real-time performance and high accuracy of monitoring ECG signal is implemented.
\end{abstract}

\section{Introduction}

Police officers of is a high stress profession for the past five years, which is mainly caused by being overworked [1]. Casualties among police officers have been increasing year by year, so it is of great practical significance to monitor the health of police officers in daily activities. Electrocardiogram (ECG) is an important signal for human body, and the vital signs of human body can be reflected in ECG signal. It can be used for analysis of heart rate variability, mental stress and fatigue detection, then sudden death of policeman from over exertion and high stress will be avoided [2].

The conductive fabric was used as the electrode to collect the ECG signal in recent years, and one of the main challenges was removing motion artifacts (MA). Paper [3] collected the ECG signals of the left and right wrist, and adaptive filter was applied on ECG signal which was obtained with textile electrodes to eliminate motion artifacts from the wrists. Castro I.D. et al [4] proposed a robust wireless system for capacitive ECG which was captured on the office chair. The motion sensors for outputting acceleration and gyroscope signals in the adaptive filters were validated in the system. Huainan Zhang et al [5] developed a new approach by using feed forward combined adaptive filter which could suppress MA and maintained low distortion for detecting the QRS waves in ECG. However, demising MA is still a great work for successful implementation of the device for monitoring ECG signal in the chest of policemen since they need to be simply, fast and computationally efficient.

This paper proposes a wearable wireless real-time detection device. First, the anti-electromagnetic interference conductive silicone is utilized as the electrode of the ECG signal. Next, highly integrated dedicated analog chips are used to capture ECG signal and a 32-bit low-power microcontroller is adopted to implement algorithms of filtering and detecting ECG signal R-wave. Finally, a variable step-size least mean square (LMS) algorithm is validated in the device, and the low-power Bluetooth 5.0 is used to achieve wireless transmission of detection systems and smart terminals. 


\section{Method}

\subsection{Hardware Implementation.}

The system block diagram is shown in Fig. 1, which includes sensor signal acquisition, signal preprocessing, data transmission and display. ADS1292 is used for collecting signal in ECG analog front end, and the sensor ADXL345 collects acceleration. Then ECG signal demising and the calculation of heart rate is implemented throng using MCU (STM32L151). The real-time transmission of the low-power Bluetooth chip nRF52832 is used to achieve real-time communication between the acquisition system and the personal domain terminal, in which the APP can display the ECG, heart rate and acceleration signals to achieve the convenience and human-computer interaction of the system for detecting ECG signals.

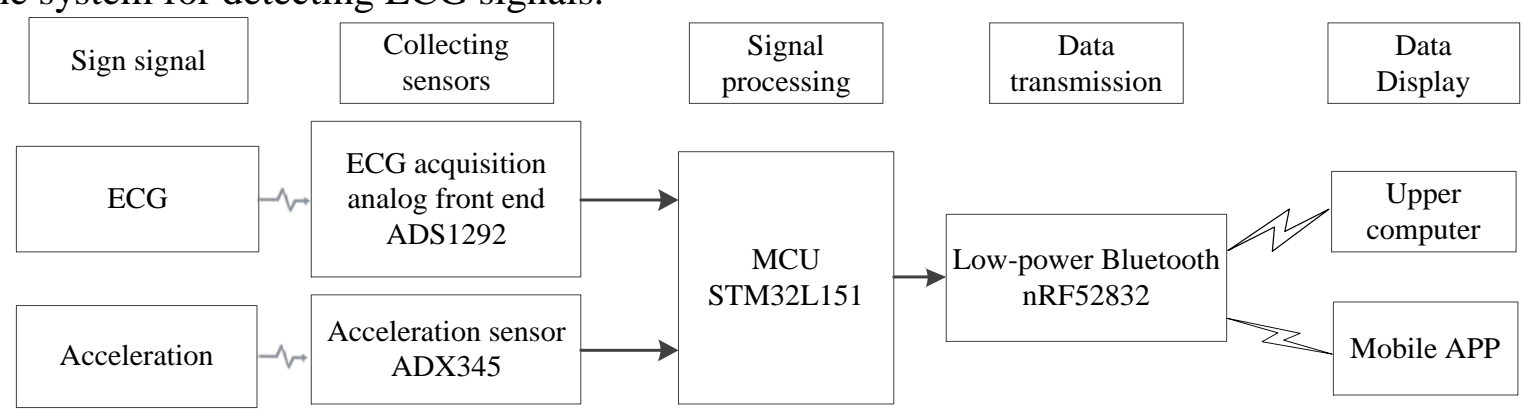

Fig. 1 Block diagram of the ECG detection system

\section{Signal Processing Algorithm}

Microcontrollers which is the core processing unit of the entire system is used for processing ECG signal in the acquisition device. Through STM321151, The signal demising and the R-wave positioning of the ECG signal are implemented and the heart rate value is calculated.

\subsection{Analysis of ECG DE Noising.}

The amplitude of the ECG signal collected by the conductive silicone electrode is weak and contains interference, including power frequency interference, myoelectric interference, MA interference, etc. Because the collected ECG signal module is sampled by using a single lead, the useful frequency is concentrated at $0.05-40 \mathrm{~Hz}$ [6]. The power line interference is filtered by the negative feedback applied by the right leg drive circuit. The electromyography interference is a high frequency component of the ECG signal. A digital FIR low-pass filter can be used to filter out the high-frequency interference.

When detecting ECG signal in motion, the body activity during the acquisition of the ECG signal should be considered [7]. The relative displacement occurs between the collecting electrode and the skin on the chest strap, thus larger low-frequency MA interference is introduced into the acquired ECG signal.

\subsection{Adaptive Filtering Algorithm.}

The ECG signal as a random signal can be filtered by the principle of coherent noise reduction with adaptive filter. The adaptive algorithm discussed in this paper uses the acceleration signal as a reference signal, which is produced by the ADXL345 accelerometer sensor, and then the filter coefficient is adjusted to automatically adapt to the current motion conditions of the body, which removes the MA interference from the ECG signal.

Block diagram of adaptive filtering is shown in Fig. 2. Noisy signals are including the clean ECG signal and MA interference, which are recorded simultaneously with the reference signal [8]. Using the optimal Wiener filtering criterion, the mean square error cost function is selected. Through the adaptive algorithm, the filter coefficient is continuously updated to achieve the minimum error cost function. 


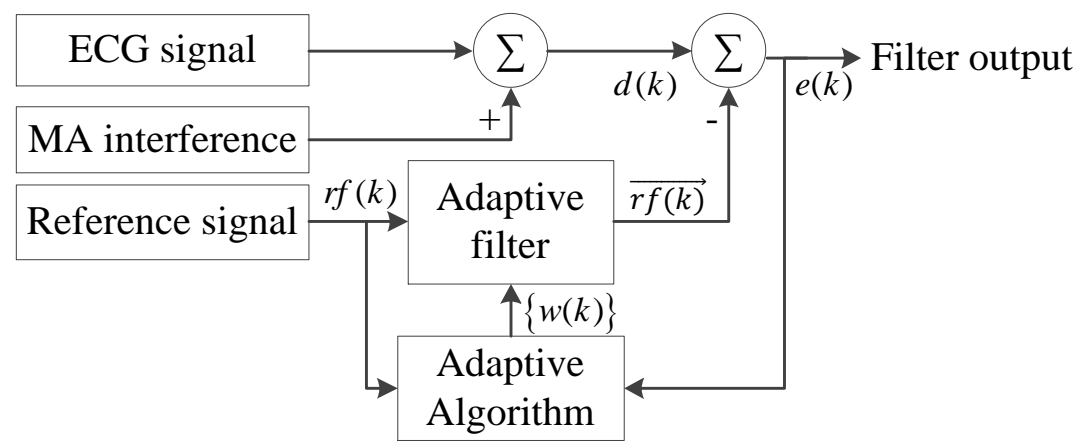

Fig. 2 Adaptive filtering block diagram

A Least Mean Square (LMS) algorithm with a variable step size is used in the ECG detection device, and an adaptive filter is calculated by the steepest descent path convergence. For M-order LMS Algorithm, the updating formula is as follows:

$$
\begin{gathered}
\boldsymbol{w}(k+1)=\boldsymbol{w}(k)+2 \mu \boldsymbol{e}(\mathrm{k}) \boldsymbol{x}(\mathrm{k}) \\
\boldsymbol{e}(\mathrm{k})=\boldsymbol{d}(\mathrm{k})-\overrightarrow{\boldsymbol{r} \boldsymbol{f}(\mathrm{k})}
\end{gathered}
$$

Where the input reference sequence is $\boldsymbol{x}(k)$ and $\mu$ is the step size of the adaptive filter which is related with the convergence rate.

In order to reduce the calculation and improve the filtering efficiency, the variable-step LMS algorithm is used to achieve the purpose of filtering out the MA interference in the single-chip microcontroller. Algorithm flow chart is shown in Fig. 3. First, the three-axis acceleration signal of the motion sensor is filtered by smoothing process. Next the maximum and minimum values found in the data intercepting a piece of acceleration signal are subtracted to obtain the variation component, thereby sensing the human motion. Then according to the size of the change component, this article divides the human body motion into three states: intense, strong and weak, which is corresponding to three different step sizes. The convergence step is appropriately changed according to the intensity of the human body, thereby realizing faster filtering of MA interference in the system.

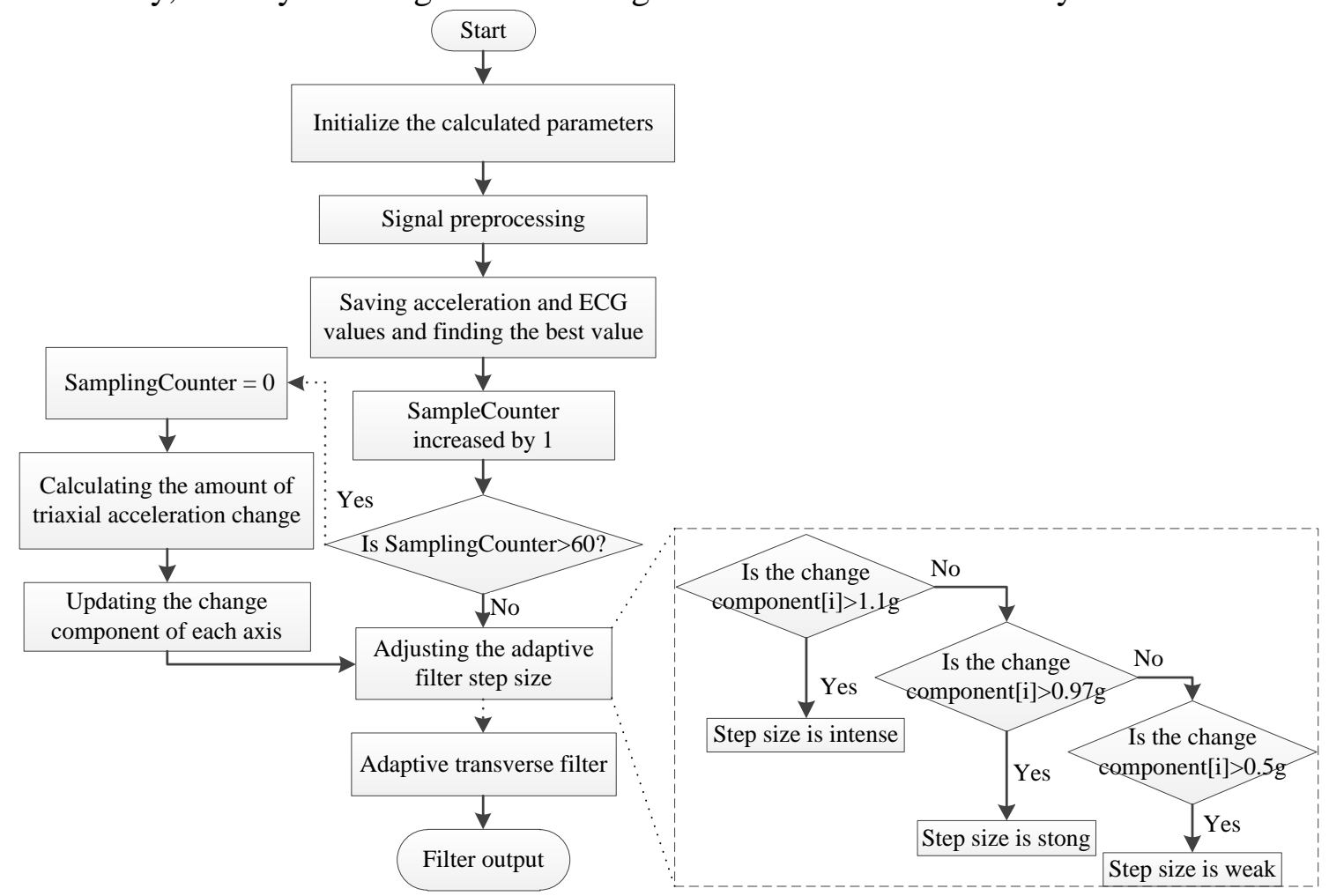

Fig. 3 Workflow of the variable-step LMS algorithm 


\subsection{Heart Rate Calculation.}

Differential threshold algorithm [9] is used to achieve real-time positioning of $\mathrm{R}$ wave after filtered ECG signal in the microcontroller system. The heart rate is calculated using the number of sample points between the two adjacent $\mathrm{R}$ waves located as a calculation parameter, and the formula is given:

$$
H R=\frac{60 \mathrm{Fs}}{R R}
$$

Where Fas is the sample rate for ECG signals, and $R R$ is the number of sample points between the two R waves.

\section{Analysis of Experimental Results}

\subsection{Detection Device.}

The appearance of the wearable ECG detection device and electrodes are shown in Fig. 4a. After the detection device is weared on the chest of the human body as shown in Fig. 4b, the ECG data and triaxle acceleration can be received on the mobile phone APP through Bluetooth. The display of signal waveform and heart rate on the Mobile APP are shown in Fig. $4 \mathrm{c}$. The detection device is $4.5 \mathrm{~cm}$ in length and $2 \mathrm{~cm}$ in width, with small size, high real-time performance and high accuracy.

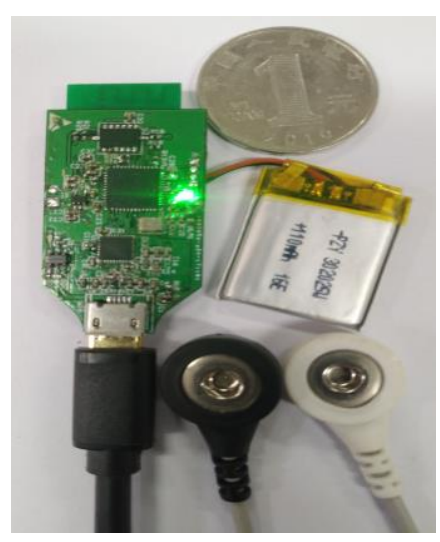

(a)

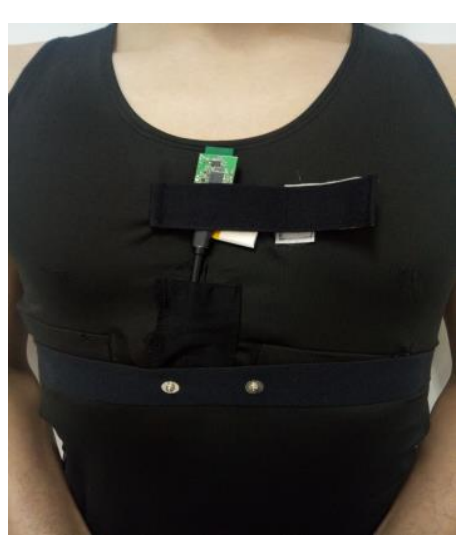

(b)

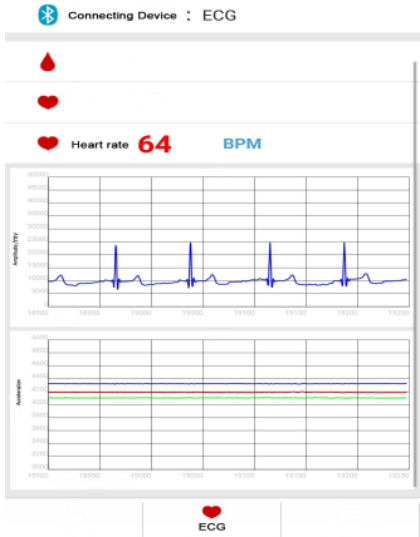

(c)

Fig. 4 The appearance of the detection device (a); Schematic diagram of the detection device worn on a human body (b); Mobile APP interface of displaying the signal waveform and heart rate(c).

\subsection{Power Consumption of the Device.}

The Keithley 2230 DC Power is utilized to provide DC power for measuring voltage and current values. When the ECG detection device is unconnected and connected to the mobile phone APP, the test results of the power consumption is shown in table 1. It can be seen that the device can work normally for more than 10 hours under the power supply of an $110 \mathrm{mAh}$ lithium cell, thus the power consumption of the device is relatively low.

Table 1. System power consumption

\begin{tabular}{ccccc}
\hline & \multicolumn{3}{c}{ Status of Bluetooth connection } \\
\cline { 2 - 5 } & \multicolumn{3}{c}{ Unconnected status } & \multicolumn{2}{c}{ Connected status } \\
\hline Voltage (V) & 3.7 & 4.2 & 3.7 & 4.2 \\
Current(mA) & 1.4 & 1.3 & 10 & 9.5 \\
Power (maw) & 5.18 & 5.46 & 37 & 39.9 \\
\hline
\end{tabular}

\subsection{The Error of Heart Rate.}

The heart rate measured by the detection device is compared with the standard medical monitor of the Mindray MEC-1000 to indicate the relative error of the system heart rate. The results are shown in table 2, in which the data is measured by 1 to 5 testers at rest and the other is measured by 6 to 10 testers in the case of body movement. It can be concluded that the heart rate value measured by the ECG detection device is within $1 \%$ in Resting ECG, and within $4 \%$ when the body is moving. 
Table 2. Heart rate comparison between test device and medical monitor

\begin{tabular}{cccc}
\hline Group number & A medical monitor $(\mathrm{bpm})$ & ECG detection device $(\mathrm{bpm})$ & Error $(\%)$ \\
\hline 1 & 63 & 63 & 0 \\
2 & 73 & 74 & 1.36 \\
3 & 72 & 71 & 1.39 \\
4 & 69 & 68 & 1.44 \\
5 & 68 & 67 & 1.47 \\
6 & 93 & 90 & 3.23 \\
7 & 84 & 82 & 2.38 \\
8 & 88 & 91 & 2.27 \\
9 & 84 & 86 & 2.38 \\
10 & 83 & 80 & 3.61 \\
\hline
\end{tabular}

\section{Summary}

In this paper,a wearable real-time ECG detection device was designed to examine the ECG signals of high-risk professional groups such as policemen and firemen. Combining triaxial acceleration sensors, the variation of triaxial acceleration is used to perceive the situation of human motion, and a variable-step LMS adaptive algorithm implemented to filter MA of ECG signal is proposed, then the $\mathrm{R}$-wave algorithm is used to calculate the heart rate. The results show that the ECG detection device has low power consumption, small size, and can display ECG signals in real time. The heart rate error during body movement is within $4 \%$.

\section{Acknowledgments}

This work was supported in part by the Research and Innovation Project of Graduate Students in Chongqing Graduate scientific Research and Innovation Project of Chongqing (CYS17239)and the Key Industry-specific Key Technology Innovations of Wearable IoT Smart Terminals (cstc2015zdcy-ztzx120002).

\section{References}

[1]. Acquadro M D, Varetto A, Zedda M, et al. Occupational stress, anxiety and coping strategies in police officers. [J]. Occupational Medicine, 2015, 65(6):466.

[2]. Vanitha L, Suresh G R. Hybrid SVM classification technique to detect mental stress in human beings using ECG signals[C]// International Conference on Advanced Computing and Communication Systems. IEEE, 2013:1-6.

[3]. Alkhidir T, Sluzek A, Yapici M K. Simple method for adaptive filtering of motion artifacts in E-textile wearable ECG sensors[C]// Engineering in Medicine and Biology Society. IEEE, 2015:3807.

[4]. Castro I D, Morariu R, Torfs T, et al. Robust wireless capacitive ECG system with adaptive signal quality and motion artifact reduction[C]// IEEE International Symposium on Medical Measurements and Applications. IEEE, 2016:1-6.

[5]. Zhang H, Zhang S, Jin Q, et al. Motion artifact suppression in ambulatory ECG with feed forward combined adaptive filter[C]// Computing in Cardiology Conference. IEEE, 2017.

[6]. Clifford G D, Assuage F, McCurry P. Advanced Methods and Tools for ECG Data Analysis[C]// Bartech House, Inc. 2006.

[7]. Chaudhary S, PA war T D, Duttagupta S. Ambulation Analysis in Wearable ECG [M]. Springer US, 2009. 
[8]. Lee W C, Yang Y S O, Key T C, et al. Adaptive reduction of motion artefact in a portable ECG system[C]// Sensors. IEEE, 2011:704-707.

[9]. Lai D, Zhang F, Wang C. A real-time QRS complex detection algorithm based on differential threshold method[C]// IEEE International Conference on Digital Signal Processing. IEEE, 2015:129-133. 